Recepción: 01 / 09/ 2018

Aceptación: 15 / 09 / 2018

Publicación: 25 / 11 / 2018

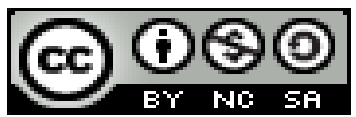

Ciencias de la salud

Artículo científico

\title{
Incisivos laterales conoideos en población escolar de niños y adolescentes comprendida entre 9 y 12 años
}

\section{Lateral conoid incisors in school population of children and adolescents between 9 and 12 years}

Incisivos laterais conóides na população escolar de crianças e adolescentes entre 9 e 12 anos

José H. Mosquera-Tayupanta ${ }^{\mathrm{I}}$

josehmosquera@hotmail.com

Tulio X. Camacho-Chávez II

tulioxcamacho@hotmail.com

Correspondencia:drtuliosavier@yahoo.com

I. Especialista de Primer Grado en Ortodoncia; Diploma Superior en Ortodoncia; Doctor en Odontología, Universidad Central de Ecuador, Quito, Ecuador.

II. Especialista de Primer Grado en Ortodoncia; Magister en Gerencia de Salud para el Desarrollo Local; Especialista en Gerencia y Planificación Estratégica de Salud; Diploma Superior de Cuarto Nivel en Desarrollo Local y Salud; Doctor en Odontología, Universidad Central de Ecuador, Quito, Ecuador. 


\section{Resumen}

Se efectuó un estudio descriptivo, transversal en niños y adolescentes de entre 9 a 12 años de la Escuela Roberto Arregui y el Instituto Tecnológico Superior Benito Juárez de la ciudad de Quito Ecuador con la finalidad de identificar la frecuencia de incisivos laterales conoideos en el periodo 2015-2016. Entre las variables analizadas sobresalieron: edad, sexo, anomalías de incisivos laterales conoideos unilateral o bilateral, anomalías de forma o tamaño que permita adaptarse al brackets. En la casuística el 5.6\% de niños presentan anomalías de dientes conoideos y entre estos un $27.0 \%$ presentan anomalías unilateral, $73.0 \%$ bilateral, la presencia respecto al sexo es del 50.0\%, además un 59.0\% presentaron adaptación al brackets. El realizar un diagnóstico oportuno de estas patologías con su correspondiente tratamiento puede evitar la presencia a futuro diastemas y mal oclusión.

Palabras Clave: Incisivos conoideos, diastemas, carillas, ortodoncia, anomalía.

\section{Abstrac}


Acoustic pollution and its incidence in population health around bus station perimeter in Jipijapa city - Ecuador

A descriptive, transversal study was conducted in children and adolescents from 9 to 12 years of the Roberto Arregui School and the Instituto Tecnologico Superior Benito Juárez of the city of QuitoEcuador in order to identify the frequency of lateral incisors conoideos in the period 2015-2016. Among the analyzed variables were: Age, sex, anomalies of lateral incisors unilateral or bilateral conoideos, anomalies of shape or size that allows to adapt to brackets. In the case of $5.6 \%$ of children have abnormalities of conoideos teeth and between these a $27.0 \%$ have unilateral anomalies, $73.0 \%$ bilateral, the presence with respect to sex is $50.0 \%$, in addition a $59.0 \%$ showed adaptation to brackets. Making a timely diagnosis of these pathologies with their corresponding treatment can avoid the presence of future diastemas and bad occlusion

Key words: Incisors conoideos, diastemas, veneers, orthodontics, anomaly.

\section{Introducción.}


Las consideraciones acercan del órgano dental arrojan que son estructuras anatómicas con particularidades individuales, su formación se basa en dos fases, la primera es donde se produce el depósito de la matriz orgánica y la segunda donde ocurre la mineralización de ésta ${ }^{1}$.

Hacemos referencia que desde la vida embrionaria se desarrolla la oclusión dentaria, es un proceso largo y complejo ya que sus condiciones no permanecen estables por factores de orden general y local que actúan sobre ella. Su tratado está lleno de eventos importantes que darán como resultado final el establecimiento de una oclusión bien establecida ${ }^{2}$.

Entre la sexta y octava semana de vida intrauterina ocurren la mayoría de las deformaciones dentales, en este periodo se produce la transformación de estructuras embrionarias importantes como son el saco dentario, papila dentaria y el órgano dentario que en el proceso de histodiferenciacion darán lugar a la formación del esmalte, dentina y cemento. La odontogenesis es el proceso de la formación del diente el mismo es continuo se inicia con la formación de la corona y termina con la formación de la raíz, la capacidad de formación de la dentina continua durante toda la vida del diente. Al referirnos a las anomalías dentales; son malformaciones congénitas de los tejidos del diente que se dan por falta o por aumento en el desarrollo de estos, estas pueden ser de forma, número, tamaño, de estructura, de posición incluso pueden provocar retraso en el cambio de los decíduos a los permanentes y en algunas ocasiones falta de desarrollo de los maxilares ${ }^{3}$.

Es importante destacar que el tamaño de los dientes está determinado genéticamente; y su disminución se debe al debilitamiento del órgano del esmalte durante el periodo de diferenciación ${ }^{4}$.

Se conceptualiza a la microdoncia como una anomalía dental determinada por una disminución del diámetro mesio-distal y cervico-incisal de la corona dental como también de la raíz, por lo que son dientes pequeños pero con anatomía adecuada, la misma se puede presentar de 
manera generalizada en los dientes o localizada (en un solo diente), siendo el incisivo lateral superior el diente en el que se presenta más modificaciones en su tamaño y forma ${ }^{5,6,7}$ Esta anomalía afecta la longitud de arco, lo que compromete la estética, ya que el diente podrá quedar en una posición incorrecta afectando la oclusión o los movimientos dentales ${ }^{8}$.

En el espacio de las observaciones anteriores es referido que la microdoncia presenta problemas que trae consecuencias en la longitud de arco, además del compromiso en la estética facial. Puede generar diastemas y migraciones dentarias indeseables que sustentan la aparición de problemas aún mayores así como estar relacionada con ausencia de segundos premolares, ausencia de terceros molares, sobre todo en mujeres, agenesia del incisivo lateral superior del lado contrario y desplazamiento de caninos hacia palatino, lo que puede producir una retención de los mismos ${ }^{9,10}$ : Es importante determinar la cantidad y la ubicación de una discrepancia en el tamaño de los dientes antes de comenzar el tratamiento ortodóntico ya que para lograr una buena intercuspidación debe existir una relación estricta entre el tamaño de los dientes superiores e inferiores así como estar en armonía con el tamaño de la arcada para que pueda darse una alineación correcta ${ }^{11}$.

Dentro de las alteraciones bucales se encuentran los incisivos laterales conoideos, por lo que son consideradas un problema de salud pública: Los que tienen forma cónica con lados que convergen o se adelgazan juntos incisalmente en vez de ser paralelos o de mostrar divergencia mesial y distal; su etiología es debido al desarrollo defectuoso del germen dentario, y se encuentra en displasia ectodérmica y otros trastornos y ocasionalmente en niños normales, fueron descritos por Grahnén como aquellos dientes en los que el ancho mesiodistalincisal de la corona es más corto que el ancho cervical ${ }^{12}$. 
La situación revelada motivó al autor a realizar esta investigación, con el objetivo de identificar en nuestro medio esta anomalía de la arcada dentaria superior con presencia de dientes laterales conoideos propiciando elegir el mejor procedimiento terapéutico para compensar la incompatibilidad entre dientes antagónicos probando la facilidad en la adaptación de los brackets en caso de tratamiento ortodóncico sobre dientes conoideos o carillas de ser el caso donde no haya un adecuado soporte radicular.

\section{Materiales y métodos.}

Se realizó un estudio descriptivo de corte transversal en la escuela Roberto Arregui e Instituto Tecnológico Superior Benito Juárez de la ciudad de Quito con la finalidad de identificar la frecuencia de incisivos laterales conoideos en población escolar de niños y adolescentes comprendida entre 9 y 12 años en el periodo 2015-2016, La población de estudio estuvo conformada por 600 niños y adolescentes de ambos sexos.

Entre las variables de interés figuraron las siguientes: edad, sexo, anomalías de incisivos laterales conoideos unilateral o bilateral, anomalías de forma o tamaño que permita adaptarse al brackets

Con respecto a los materiales usados fueron:

- Compás de puntas romas.

- Regla.

- Brackets de la pieza Nro. 11 o 21.

- Porta brackets.

- Espejos exploradores. 
- Pinzaalgodonera.

- Cámara digital.

- Espejos intra orales.

- Separadores bucales.

A los niños se los examinó sentados en el sillón odontológico bajo luz natural y artificial. En el examen clínico se centro en la arcada superior haciéndose una medición de los incisivos laterales utilizando el compás de punta roma, se colocaron en los bordes laterales de los dientes mencionados y luego se medio con la ayuda de una regla la distancia entre las puntas para determinar su tamaño para con ello reconocer incisivos laterales conoideos. Se tendrá en cuenta la adaptabilidad del brackets para el tratamiento de la desarmonía entre los tamaños dentarios de la arcada superior con presencia de dientes laterales conoideos y la arcada inferior.

Para la realización de la investigación se tuvo en cuenta el consentimiento informado y a los pacientes objeto de estudio se les explicó en qué consistía dicha investigación, la confidencialidad de los datos obtenidos y que no se produciría daño a su integridad, para lo cual se siguieron los principios de la bioética, además del respeto y la autonomía de la persona; si el paciente no deseaba participar, no se incluiría en el estudio.

Para la recolección de datos en este estudio se utilizó el formulario odontológico de la historia clínica del Ministerio de Salud Pública, en el que se registró las anomalías incisivos laterales conoideos que presentaba cada paciente así como la no existencia de esta anomalía.

Los datos obtenidos se recolectaron en una hoja de Excel y se analizaron a través de estadística descriptiva utilizando medidas de resumen. 


\section{Resultados.}

La tabla 1 muestra que de un total de 600 niños examinados el $5.6 \%$ (34) presentaron dientes laterales superiores conoideos en el cual el objetivo principal es conocer si se logra la adaptabilidad de los brackets para un posterior tratamiento ortodóncico, la colocación de carillas o prostodoncia según corresponda.

Tabla 1. Pacientes que presentaron incisivos laterales conoideos.

\begin{tabular}{|c|c|c|}
\hline $\begin{array}{c}\text { Pacientes que } \\
\text { presentaron incisivos } \\
\text { laterales conoideos }\end{array}$ & No & \% \\
\hline $\mathrm{Si}$ & 34 & 5.6 \\
\hline $\mathrm{No}$ & 566 & 94.3 \\
\hline
\end{tabular}

Véase en la tabla 2 refiriéndose a un estado comparativo de la variable sexo resulto de 34 pacientes examinados correspondió el $50 \%$ a cada sexo.

Tabla 2 Presencia de incisivos laterales conoideos de los 34 casos encontrados por sexo.

\begin{tabular}{|c|c|c|}
\hline Sexo & No & \% \\
\hline Masculino & 17 & 50.0 \\
\hline Femenino & 17 & 50.0 \\
\hline TOTAL & 34 & $100 \%$ \\
\hline
\end{tabular}

Al analizar la tabla 3 se hallo un predominio de dientes laterales conoideos bilaterales $(73.5$

$\%)$ 
Tabla 3. Presencia de dientes laterales conoideos unilaterales y bilaterales.

\begin{tabular}{|c|c|c|}
\hline Dientes conoideos & No & \% \\
\hline Unilaterales & 9 & 26.4 \\
\hline Bilaterales & 25 & 73.5 \\
\hline Total & 34 & 100 \\
\hline
\end{tabular}

En esta serie (tabla 4) prevaleció la adaptación del brackets a la cara vestibular del incisivo lateral con $59.0 \%$

Tabla 4 Adaptación al brackets del incisivo lateral conoideo por su cara vestibular, para tratamiento ortodontico.

\begin{tabular}{|l|l|l|}
\hline Adaptación del brackets a la cara vestibular & No & $\%$ \\
\hline del incisivo lateral & 20 & 59.0 \\
\hline $\mathrm{Si}$ & 14 & 41.0 \\
\hline No & & \\
\hline
\end{tabular}

\section{Discusión.}


La población de estudio no representa una proyección a la población general, sin embargo epidemiológicamente presenta relevancia significativa. Referencias aportadas por bibliografías revisadas en sus escritos pronuncian que en nuestro país existen escasos estudios que demuestren la prevalencia de incisivos laterales conoideos a temprana edad, situación que en un futuro afecta la estética a esta población al no tener un diagnóstico oportuno.

Los factores como sexo, raza, ambiente, herencia, cambios seculares y simetría bilateral influyen en el tamaño y forma del diente. La microdoncia puede producir una alteración en el espacio de la arcada, exfoliación tardía del diente primario o una erupción tardía del diente permanente ${ }^{13}$.

Común y frecuentemente existe en la población la presencia de diastemas o separación entre dientes. También encontramos múltiples variaciones de la forma y/o tamaño de los mismos, como por ejemplo los dientes conoides. Según Masías Percca ${ }^{14}$ reporto en su estudio que los dientes en clavija fueron los de mayor prevalencia, no siendo concurrente con lo encontrado en esta investigación.

En este mismo orden y dirección diversos investigaciones plantean la prevalencia de los dientes conoides ha sido reportada variando de 0,6 a 9,9\%. Describe Santos Peñaloza según un meta-análisis realizado por Hua a nivel mundial, casi una de cada 55 personas $(1,88 \%)$ es afectada por laterales conoides, en nuestro estudio tenemos una prevalencia de $5.6 \%$ de incisivos laterales conoideos estando dentro del rango a nivel mundial. El sexo femenino representa 1,35 veces más propensas que el sexo masculino, no coincidiendo con los resultados de la investigación. Los laterales conoides tanto unilaterales como bilaterales ocurren con la misma frecuencia, pero cuando ocurre unilateralmente existe cierta predisposición en el lado izquierdo. Granat y Chappelle, referido 
por Aguirre y Noborokawa reseñaron que en la evolución de una especie un diente es reducido en volumen y se vuelve cónico antes de desaparecer. Además, los factores genéticos y ambientales son presuntamente responsables de la falta de dientes y tamaños reducidos de éstos ${ }^{15}$.

Como se ha explicado los dientes conoides generalmente afectan a los incisivos laterales superiores, esta situación es muy favorable para las carillas de cerámica ya que la preparación es prácticamente nula y la agresión al tejido dentario mínima, por lo que se considera una muy buena solución terapéutica, que armoniza el tamaño dentario y estabiliza la oclusión. En este tipo de malformación dentaria, las carillas de porcelana han de ser muy amplias para cerrar los espacios interproximales. De esta forma, en muchos casos vienen a tener la forma casi de una corona de recubrimiento total con una terminación cervical en filo de cuchillo ${ }^{16}$. Experimentados especialistas plantean que los brackets son un tipo de ortodoncia fija que se adhieren a la superficie de los dientes y sobre los cuales se coloca un fino alambre y además unas gomas, para conseguir una sonrisa perfecta recolocando los dientes mal posicionados, así como corregir entre otros, problemas de oclusión. En esta serie predomino la adaptabilidad del brakets presenta un $59.0 \%$ lo que facilitaría un tratamiento ortodontico dependiendo del soporte radicular. Estudios realizados en una población de México reflejo un alto porcentaje de microdoncia de ILS de acuerdo a los criterios de Binder y Cohen lo cual debe considerarse en la planeación del tratamiento ortodóncico ${ }^{5}$.

Se concluye, que casi todos los niños de la serie presentaron incisivos laterales conoideos bilateral, igualmente la mayoría mostraron adaptación al brackets dato relevante para elegir un tratamiento ortodontitco. El realizar un diagnóstico oportuno de estas patologías con su correspondiente tratamiento puede evitar la presencia a futuro diastemas y mal oclusión. 


\section{Bibliografía.}

1. Pier-Domenico B, Jiménez H. Prevalencia de microdoncias mediante estudios radiográficos en pacientes del postgrado de ortopedia dentofacial y ortodoncia. Facultad de odontología Universidad de Carabobo. ODOUS Científica. 2006; 7(1):37-45.

2. Torres Carvajal M. Desarrollo de la dentición. La dentición primaria. Revista Latinoamericana de Ortodoncia y Odontopediatria. 2009 [citado 2018 Ago 30]. Disponible en: https://www.ortodoncia.ws/publicaciones/2009/art-23/

3. Mursulí Sosa M, Rodríguez Bello H, Landa Mendoza D, Hernández M . Anomalías dentales. Gaceta Médica Espirituana 2009 [citado 2018 Ago 30]; ; 8(1), Disponible en:http://www.bvs.sld.cu/revistas/gme/pub/vol.8.\%281\%29_12/p12.html

4. Jiménez M. Odontopediatría en atención primaria. España. Ed. Vértice; 2007:83-84.

5. Gómez-Fernández D, Rivas-Gutiérrez R, Gutiérrez-Rojo JF. Prevalencia de microdoncia de incisivos laterales superiores en una población mexicana. Revista CES Odontología. 2013; 26(2) 67-73.

6. Soto-Llanos L, Calero J. Anomalías dentales en pacientes que asisten a la consulta particular e institucional en la ciudad de Cali 2009-2010. Rev Estomat. 2010; 18(1): 17-23.

7. Carmona L. Diente cónico: presentación de dos casos. Rev Méd Risaralda. 2014; 20 (2):125128.

8. García-Castillo M, Tavira S. Enfoque multidisciplinario para el manejo de espacios en presencia de microdoncia y retención de un incisivo superior: reporte de un caso. Revista Mexicana de Ortodoncia. 2016; 4 (1): 56-61.

9. Utrell-Torrent J. Manual de Ortodoncia. Etiopatogenia de las maloclusiones. Barcelona. UBe; 2011:193. 
10. Gómez-Fernández D, Rivas-Gutiérrez R, Gutiérrez-Rojo J . . Prevalencia de microdoncia de incisivos laterales superiores en una población mexicana. Revista CES Odontología. 2013; 26(2)

11. Kubodera T, Zárate C, Lara E, Montiel M, Pereyra G, Centeno C. Dimensiones coronales mesiodistales en la dentición permanente de mexicanos. Revista ADM. 2008; 65 (3):141-149.

12. Grahnén H. Hypodontia in thepermanentdentition: a clinical and geneticalinvestigation. Odont Rev. 1956; 7:1-100.

13. Cruz-López María Fernanda, Gutiérrez-Rojo Jaime Fabián, Moreno Moreno José Javier, Rojas-García Alma Rosa, Gutiérrez-Villaseñor Jaime. Microdoncia de incisivos laterales maxilares en mujeres y hombres. Rev Latinoamericana de ortodoncia y odontopediatria 2016 [citado 2018 julio 20] .Disponible en :https://www.ortodoncia.ws/publicaciones/2016/art-47/

14. Masias Percca R. Prevalencia de anomalías dentales en forma, Tamaño y número en pacientes de 3 a 6 años de edad Con dentición decidua que asistieron a la clínica Docente de la Upc Durante los años 2012 a 2014. 2015 [citado 2018 julio 20] .Disponible en :https://core.ac.uk/download/pdf/54246974.pdf

15. Santos Peñaloza D. Alteraciones de desarrollo dental en alumnos con síndrome de down de 6 a 25 años de edad del c.e. especial unámonos, Arequipa 2015. Tesis presentada para optar por el título de Cirujano mdentista. [citado 2018 julio 20] .Disponible en: https://core.ac.uk/download/pdf/54220121.pdf.

16. Rábago-Vega José de, Tello-Rodríguez Ana Isabel. Carillas de porcelana como solución estética en dientes anteriores: informe de doce casos. RCOE. 2005 [citado 2018 Nov 15]; 10 ( 3

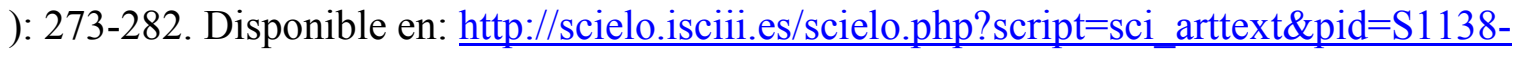
$\underline{123 \times 2005000300003 \& \operatorname{lng}=\mathrm{es}}$ 


\section{ANEXOS}

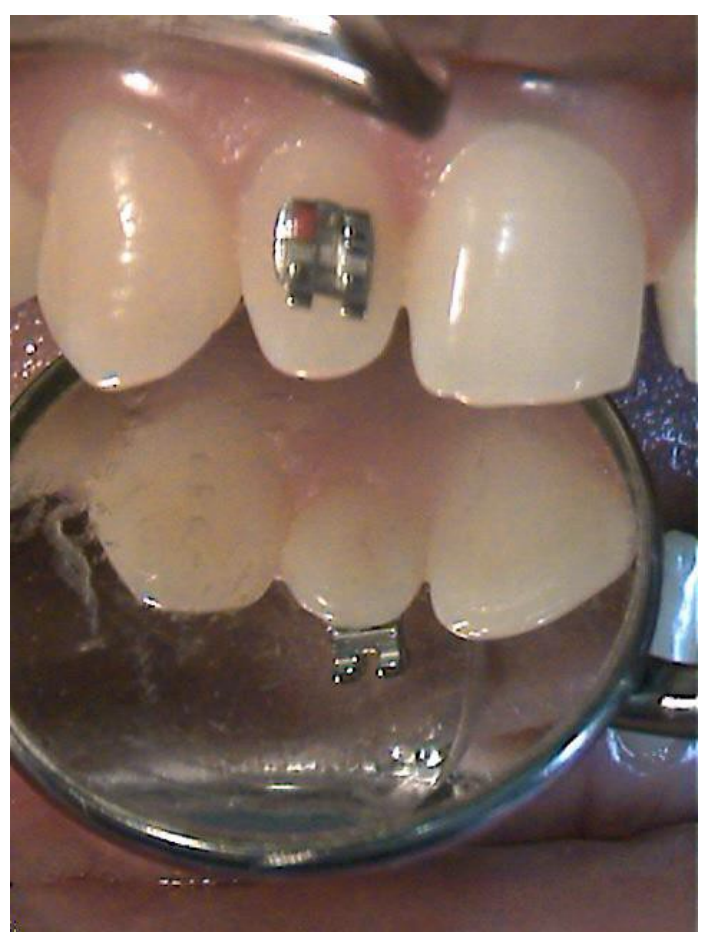

Gráfico (a) Pieza dental \# 12 conoidea con facilidad de colocación deBraket. Vista palatina.

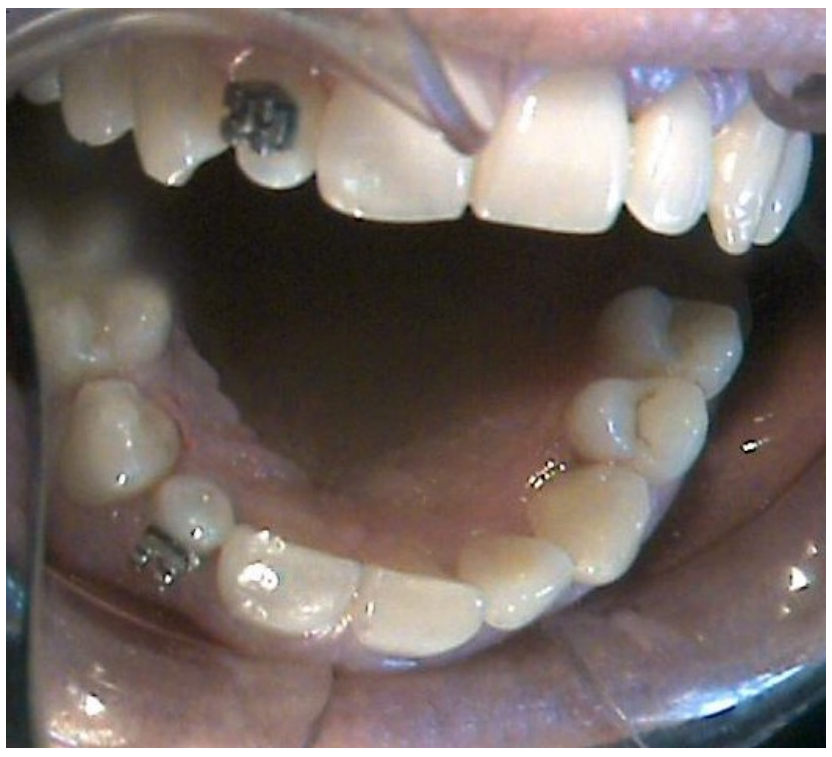

Gráfico (b) Presencia unilateral de dientes conoideos Pieza dental \# 12. 


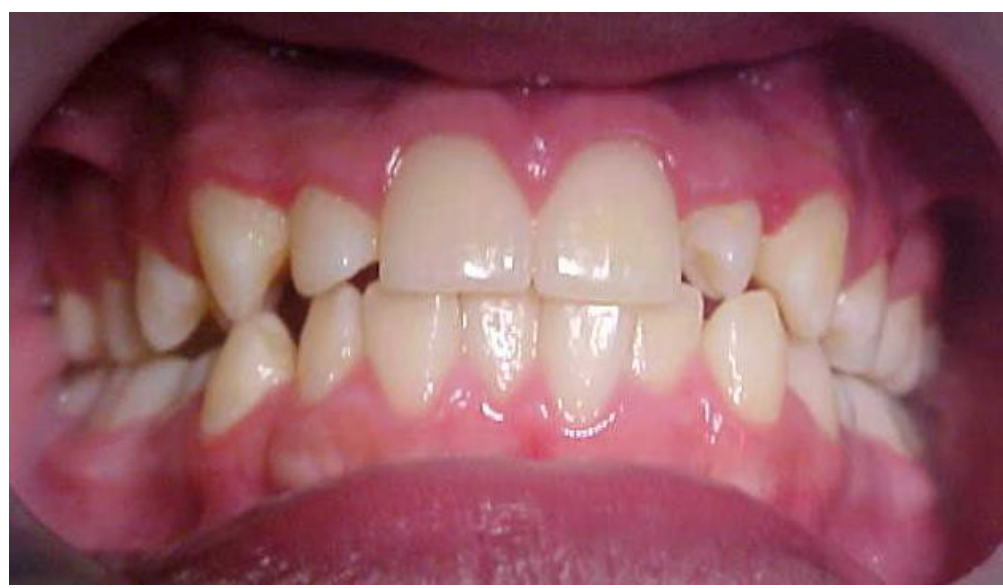

Gráfico (c) Presencia de incisivos lateralesconoideos, bilateral 12 - 22. No adaptable para braket.

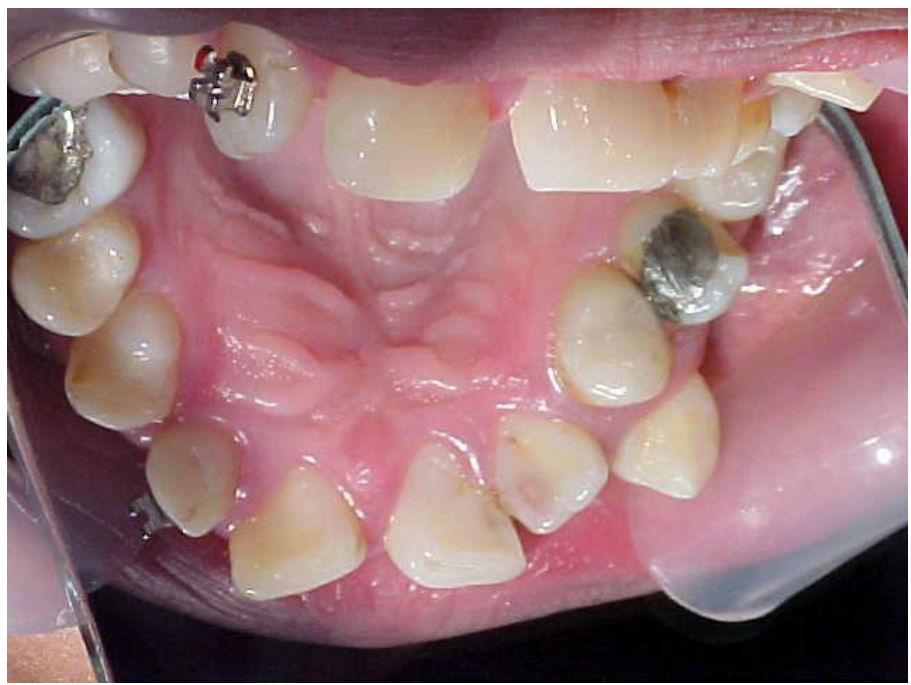

Gráfico:(d) Incisivo latera \# 12 conoideo unilateral con facilidad de tratamiento ortodontico. 